\title{
A GENERALIZED BANACH-STONE THEOREM
}

\section{MICHAEL CAMBERN ${ }^{1}$}

If $X$ is a locally compact Hausdorff space, we denote by $C_{0}(X)$ the Banach space of continuous complex-valued functions on $X$ which are zero at infinity, with norm given by

$$
\|f\|=\sup _{x \in X}|f(x)|, \quad f \in C_{0}(X) .
$$

(We recall that if $X$ is compact, then $C_{0}(X)$ consists of all continuous complex-valued functions on $X$.) The well known Banach-Stone theorem states that if $X$ and $Y$ are locally compact Hausdorff spaces, and if $C_{0}(X)$ and $C_{0}(Y)$ are isometrically isomorphic, then $X$ and $Y$ are homeomorphic. The purpose of this article is to show that, with the additional hypothesis of first countability (which may well be unessential), the conclusion of the theorem remains true for a class of mappings somewhat more general than isometries.

We wish to prove the following:

THEOREM. If $X$ and $Y$ are first countable, locally compact Hausdorff spaces, and $\phi$ is a continuous, norm-increasing linear isomorphism of $C_{0}(X)$ onto $C_{0}(Y)$ with bound strictly less than two,

$$
\|f\| \leqq\|\phi(f)\| \leqq\|\phi\|\|f\|, \quad f \in C_{0}(X),\|\phi\|<2,
$$

then $X$ and $Y$ are homeomorphic.

In $[1$, p. 242] Banach defines, for two Banach spaces $E$ and $F$, the number $(E, F)$ by

$$
(E, F)=\text { g.l.b. }\left\{\log \left(\|\phi\|\left\|\phi^{-1}\right\|\right)\right\},
$$

where $\phi$ runs through all isomorphisms of $E$ onto $F$. He calls $E$ and $F$ "nearly isometric" if $(E, F)=0$, and asks whether the "near isometry" of two infinite-dimensional Banach spaces may imply that they are isometric. In particular, he inquires whether the spaces $c$ of convergent sequences (continuous functions on the one-point compactification of the positive integers) and $c_{0}$ of sequences convergent to zero (functions continuous on the positive integers and zero at infinity), which are not isometric, are nearly isometric. Now if $\phi$ is any continuous isomorphism of one Banach space on to another, we may, of course, obtain a norm-increasing isomorphism $\phi^{\prime}$ by defining $\phi^{\prime}$ to

Received by the editors June 2, 1965.

1 National Science Foundation postdoctoral fellow. 
be equal to $\left\|\phi^{-1}\right\| \phi$. Thus the above theorem shows that $\left(c, c_{0}\right) \geqq \log 2$. More generally, it provides the following:

Corollary. For the class of Banach spaces $C_{0}(X)$, with $X$ a first countable locally compact Hausdorff space, the notions of "isometry" and "near isometry" are equivalent.

Before beginning the proof of the theorem, we wish to discuss briefly the notation and method of proof to be employed. We recall that if $f^{*}$ is any element of $C_{0}(X)^{*}$, then by the Riesz representation theorem [2, p. 266] there exists a unique finite complex-valued regular Borel measure $\mu$ on $X$ such that $f^{*}(f)=\int f d \mu$ for all $f \in C_{0}(X)$, with $\left\|f^{*}\right\|=|\mu|(X)$, where $|\mu|$ denotes the total variation of $\mu$. We will henceforth make this identification of continuous linear functionals and measures in our notation, applying, for example, the adjoint mapping $\phi^{*}$ to measures $\mu$ of the proper type on $Y$, and writing $\mu \in C_{0}(Y)^{*}$. Moreover, for any point $x \in X$ (resp. $y \in Y$ ) we will denote by $\mu_{x}$ (resp. $\mu_{y}$ ) the positive unit mass concentrated at the point $x$ (resp. $y$ ).

One of the standard proofs of the Banach-Stone theorem depends on a characterization of the extreme points of the unit ball of $C_{0}(X)^{*}$ -these points are precisely those measures of the form $\lambda \mu_{x}$, for $x \in X$ and $\lambda$ a complex number of modulus one. If $\phi$ maps $C_{0}(X)$ isometrically onto $C_{0}(Y)$, then $\phi^{*}$ maps the set of extreme points of the unit ball of $C_{0}(Y)^{*}$ onto the corresponding set in $C_{0}(X)^{*}$. Hence, for each $y \in Y$, there exists an $x \in X$ and a complex number $\lambda$ with $|\lambda|=1$, such that

$$
\phi^{*} \mu_{y}=\lambda \mu_{x}
$$

and the homeomorphism between $X$ and $Y$ is deduced from the correspondence between $x$ and $y$ defined by (1). Here we will be dealing with isomorphisms $\phi$ which need not be isometric, and hence $\phi^{*}$ need not map an extreme point into an extreme point. What we show is that if $\|\phi\|$ is less than 2 , then for each $y \in Y, \phi^{*} \mu_{\nu}$ is "close" to a unique extreme point of the unit ball of $C_{0}(X)^{*}$. We now make this precise.

PROOF OF THE THEOREM. Let $M$ be a real number with $\|\phi\|<2 M<2$, and let $M^{\prime}=\|\phi\|-M$. For any $x \in X$ and $y \in Y, \phi^{*} \mu_{y}$ may be written uniquely as $\alpha \mu_{x}+\mu$, where $\alpha$ is a complex number (which may, of course, be zero) and $\mu \in C_{0}(X)^{*}$ is such that $\mu(\{x\})=0$. We denote by $Y_{1}$ the set of all $y \in Y$ for which there exists an $x \in X$ such that

$$
\phi^{*} \mu_{y}=\alpha \mu_{x}+\mu, \quad|\alpha|>M, \mu(\{x\})=0 .
$$


Note that because $\mu_{x}$ and $\mu$ are concentrated on disjoint subsets of $X$, $\left\|\phi^{*} \mu_{\nu}\right\|=|\alpha|+\|\mu\|$. Since $\left\|\mu_{y}\right\|=1$ and $\left\|\phi^{*}\right\|=\|\phi\|$, we have $\|\mu\|<M^{\prime}$ $<M$, which insures that at most one $x \in X$ may be associated by (2) with any given $y \in Y$. Thus the mapping $\rho$ of $Y_{1}$ to $X$ determined by $\rho(y)=x$, where $x$ is associated with $y$ by (2), is indeed well defined.

Next we define $N$ by $N=1 / 2 M$, and $N^{\prime}$ by $N^{\prime}=1-N$. We then denote by $X_{1}$ the subset of $X$ consisting of all points $x$ for which there exists a $y \in Y$ with

$$
\phi^{*-1} \mu_{x}=\beta \mu_{y}+\mu, \quad|\beta|>N, \quad \mu(\{y\})=0 .
$$

Since $\left\|\phi^{*-1}\right\|=\left\|\phi^{-1}\right\| \leqq 1$, here we have $\|\mu\|<N^{\prime}<N$, and we obtain a well defined mapping $\tau$ of $X_{1}$ to $Y$ by setting, for $x \in X_{1}, \tau(x)=y$, where $y$ is associated with $x$ by (3). The proof of the theorem is now completed by means of three propositions.

Proposition 1. $\rho$ (resp. $\tau$ ) is a mapping of $Y_{1}$ (resp. $X_{1}$ ) onto $X$ (resp. Y).

Proof. Let $x$ be any point of $X$ and let $\left\{U_{n}: n=1,2, \cdots\right\}$ be a neighborhood basis at $x$, with $U_{n+1} \subseteq U_{n}$ for all $n$. For each $n$, choose a function $f_{x, n} \in C_{0}(X)$, with $f_{x, n}(x)=\left\|f_{x, n}\right\|=1$, and $f_{x, n}\left(x^{\prime}\right)=0$ for $x^{\prime} \in X-U_{n}$ (i.e., we choose a bounded sequence of functions converging pointwise to the characteristic function of $\{x\})$. Then $\lim _{n} \int f_{x, n} d \mu$ exists for all $\mu \in C_{0}(X)^{*}$, and hence $\lim _{n} \int \phi\left(f_{x, n}\right) d \mu$ exists for all $\mu \in C_{0}(Y)^{*}$. In particular, $\lim _{n} \int \phi\left(f_{x, n}\right) d \mu_{y}=\lim _{n}\left(\phi\left(f_{x, n}\right)\right)(y)$ exists for all $y \in Y$.

We claim there is at least one $y \in Y$ such that $\lim _{n}\left|\left(\phi\left(f_{x, n}\right)\right)(y)\right|$ $>M$. For if not, since the moduli $\left|\phi\left(f_{x, n}\right)\right|$ are uniformly bounded by 2, we would obtain

$$
\begin{aligned}
1 & =\lim _{n} \int f_{x, n} d \mu_{x}=\lim _{n} \int \phi\left(f_{x, n}\right) d\left(\phi^{*-1} \mu_{x}\right) \\
& =\int\left(\lim _{n} \phi\left(f_{x, n}\right)\right) d\left(\phi^{*-1} \mu_{x}\right) \leqq \int\left|\left(\lim _{n} \phi\left(f_{x, n}\right)\right)\right| d\left|\phi^{*-1} \mu_{x}\right| \leqq M,
\end{aligned}
$$

which is absurd. Hence fixing such a $y$, and writing $\phi^{*} \mu_{y}=\alpha \mu_{x}+\mu$, $\alpha$ complex and $\mu(\{x\})=0$, we have

$$
\begin{aligned}
\lim _{n}\left(\phi\left(f_{x, n}\right)\right)(y) & =\lim _{n} \int \phi\left(f_{x, n}\right) d \mu_{y}=\lim _{n} \int f_{x, n} d\left(\phi^{*} \mu_{y}\right) \\
& =\lim _{n} \int f_{x, n} d\left(\alpha \mu_{x}\right)=\alpha .
\end{aligned}
$$


Thus $|\alpha|>M, y \in Y_{1}$, and $\rho(y)=x$. Moreover, since $x$ was an arbitrary element of $X, \rho$ maps $Y_{1}$ on to $X$.

Similarly, we see that if $y$ is any point of $Y$, and $g_{v, n}$ is a uniformly bounded sequence of elements of $C_{0}(Y)$ converging pointwise to the characteristic function of $\{y\}$, there must exist at least one $x$ in $X$ with $\lim _{n}\left|\left(\phi^{-1}\left(g_{y, n}\right)\right)(x)\right|>N$. For such an $x$ we have $\phi^{*-1} \mu_{x}=\beta \mu_{y}+\mu$, $\beta$ complex, $|\beta|>N, \mu(\{y\})=0$, and the statements about $X_{1}$ and $\tau$ follow.

Proposition 2. If $y \in Y_{1}$, and $\rho(y)=x$, then $x \in X_{1}$ and $\tau(x)=y$.

PROOF. Let $y$ belong to $Y_{1}$ and let $g_{y, n}$ be a sequence of elements of $C_{0}(Y)$ with uniformly bounded moduli, converging pointwise to the characteristic function of $\{y\}$. Let $\rho(y)=x$, and suppose that either $x$ is not an element of $X_{1}$, or that $x$ belongs to $X_{1}$ but $\tau(x) \neq y$. Either supposition leads to the conclusion that $\lim _{n}\left|\left(\phi^{-1}\left(g_{y, n}\right)\right)(x)\right| \leqq N$. We might then define $P$ by

$$
P=\sup _{x^{\prime} \in X}\left|\lim _{n}\left(\phi^{-1}\left(g_{y, n}\right)\right)\left(x^{\prime}\right)\right|,
$$

and choose $x^{\prime} \in X$ such that

$$
\lim _{n}\left|\left(\phi^{-1}\left(g_{y, n}\right)\right)\left(x^{\prime}\right)\right|>\max \left\{N, M^{\prime} P / M\right\} .
$$

Now $x^{\prime} \neq x$, so that there exists a $y^{\prime} \in Y_{1}, y^{\prime} \neq y$, with $\rho\left(y^{\prime}\right)=x^{\prime}$. That is to say, $\phi^{*} \mu_{y^{\prime}}=\alpha \mu_{x^{\prime}}+\mu$, with $|\alpha|>M, \mu\left(\left\{x^{\prime}\right\}\right)=0$, and $\|\mu\|<M^{\prime}$. We would then have

$$
\begin{aligned}
0 & =\lim _{n} \int g_{y, n} d \mu_{y^{\prime}}=\lim _{n} \int \phi^{-1}\left(g_{y, n}\right) d\left(\phi^{*} \mu_{y^{\prime}}\right) \\
& =\lim _{n} \int \phi^{-1}\left(g_{y, n}\right) d\left(\alpha \mu_{x^{\prime}}\right)+\lim _{n} \int \phi^{-1}\left(g_{y, n}\right) d \mu .
\end{aligned}
$$

But the modulus of the first term on the right is greater than $M^{\prime} P$, while the modulus of the second term is less than $M^{\prime} P$, and this contradiction completes the proof of the proposition.

Proposition 3. $Y_{1}=Y$, and $\rho$ is a homeomorphism of $Y$ onto $X$.

Proof. The previous proposition implies that $X=\rho_{i}\left(Y_{1}\right) \subseteq X_{1}$. It also shows that $Y=\tau\left(X_{1}\right) \subseteq Y_{1}$. (For $\rho$ maps $Y_{1}$ onto $X$; hence given $x \in X_{1} \subseteq X$, there exists a $y \in Y_{1}$ with $\rho(y)=x$. By Proposition 2, $\tau(x)=y \in Y_{1}$.) Thus $\rho$ maps $Y$ onto $X, \rho$ is injective since $\tau$ is a function, and $\tau=\rho^{-1}$. 
We next show that $\tau$ is a closed mapping. Suppose that $F$ is a closed subset of $X$. If $y \notin \tau(F)$, then $\rho(y)=x \notin F$, and $\phi^{*} \mu_{y}=\alpha \mu_{x}+\mu$, with $|\alpha|>M, \mu(\{x\})=0$, and $\|\mu\|<M^{\prime}<M$. Since $X-F$ is open and $\mu$ is regular, there exists $f_{x} \in C_{0}(X)$ with $\left\|f_{x}\right\|=f_{x}(x)=1, f_{x}\left(x^{\prime}\right)=0$ for $x^{\prime} \in F$, and $\int\left|f_{x}\right| d|\mu|<|\alpha|-M$. This means that

$$
\begin{aligned}
\left|\left(\phi\left(f_{x}\right)\right)(y)\right| & =\left|\int \phi\left(f_{x}\right) d \mu_{y}\right|=\left|\int f_{x} d\left(\phi^{*} \mu_{y}\right)\right| \\
= & \left|\int f_{x} d\left(\alpha \mu_{x}\right)+\int f_{x} d \mu\right| \geqq\left|\int f_{x} d\left(\alpha \mu_{x}\right)\right| \\
& -\left|\int f_{x} d \mu\right|>M .
\end{aligned}
$$

But for $y^{\prime} \in \tau(F), \phi^{*} \mu_{y^{\prime}}=\alpha^{\prime} \mu_{x^{\prime}}+\mu^{\prime}$, with $x^{\prime} \in F, \mu\left(\left\{x^{\prime}\right\}\right)=0$, and $\left\|\mu^{\prime}\right\|<M^{\prime}$, so that

$$
\begin{aligned}
\left|\left(\phi\left(f_{x}\right)\right)\left(y^{\prime}\right)\right| & =\left|\int \phi\left(f_{x}\right) d \mu_{y^{\prime}}\right|=\left|\int f_{x} d\left(\phi^{*} \mu_{y^{\prime}}\right)\right| \\
& =\left|\int f_{x} d\left(\alpha^{\prime} \mu_{x^{\prime}}\right)+\int f_{x} d \mu^{\prime}\right| \leqq \int\left|f_{x}\right| d\left|\mu^{\prime}\right|<M^{\prime}<M .
\end{aligned}
$$

Hence, if we choose such a function $f_{x}$ for each $x=\rho(y)$, with $y \in Y$ $-\tau(F)$, then

$$
\tau(F)=\bigcap_{x \in X-F}\left\{y:\left|\left(\phi\left(f_{x}\right)\right)(y)\right| \leqq M\right\},
$$

a closed set. Therefore $\tau=\rho^{-1}$ is a closed mapping and $\rho$ is continuous. By an analogous argument, $\rho^{-1}$ is continuous, and thus $\rho$ is a homeomorphism.

REMARK. I have recently been informed that the case $\left(c, c_{0}\right)$ $\geqq \log 2$ was previously proved, but not published, by A. Pełczyński.

\section{REFERENCES}

1. S. Banach, Théorie des opérations linéaires, Monografie Matematyczne, Warsaw, 1933.

2. W. Rudin, Fourier analysis on groups, Interscience, New York, 1962.

Université de Paris, Orsay, France 